\title{
Sexual Arousal and Desire: Interrelations and Responses to Three Modalities of Sexual Stimuli
}

\author{
Katherine L. Goldey, $\mathrm{MS}^{\star}$ and Sari M. van Anders, $\mathrm{PhD}^{\dagger}$ \\ *Department of Psychology, University of Michigan, Ann Arbor, MI, USA; †Departments of Psychology \& Women's \\ Studies, Program in Neuroscience, Reproductive Sciences Program, University of Michigan, Ann Arbor, MI, USA \\ DOI: 10.1111/j.1743-6109.2012.02845.x
}

\begin{abstract}
A B S T R A C T
Introduction. Traditionally, sexual desire is understood to occur spontaneously, but more recent models propose that desire responds to sexual stimuli.

Aims. To experimentally assess whether sexual stimuli increased sexual desire; to compare how sexual arousal and desire responded to three modalities of sexual stimuli: erotic story, unstructured fantasy, and the Imagined Social Situation Exercise (ISSE).

Methods. In an online study, participants (128 women, 98 men) were randomly assigned to one of four arousal conditions (ISSE, story, fantasy, or neutral), and then completed desire measures. In the ISSE, participants imagined and wrote about a positive sexual encounter with a self-defined attractive person.

Main Outcome Measures. Sexual arousal (perceived genital, psychological, and perceived autonomic), anxiety, positive and negative affect, and state sexual desire via self-report measures pre- and post-condition; "trait" desire via the Sexual Desire Inventory post-condition.

Results. All three sexual conditions significantly increased sexual arousal and positive affect compared with the neutral condition, with trends for higher arousal to unstructured fantasy than the ISSE or story conditions. Sexual conditions significantly increased scores on state measures of sexual desire. In addition, sexual context influenced measurement of "trait" solitary sexual desire in women, such that women reported significantly higher trait desire after the neutral and ISSE conditions vs. fantasy.

Conclusion. Results highlight the responsiveness of sexual desire, problems with measurement of desire as a long-term trait, trade-offs of using the ISSE and other stimuli in sexuality research, and the need to address context in discussions of women's and men's desire. Goldey KL and van Anders SM. Sexual arousal and desire: Interrelations and responses to three modalities of sexual stimuli. J Sex Med 2012;9:2315-2329.
\end{abstract}

Key Words. Sexual Arousal; Sexual Desire; Fantasy; Erotica; Gender; Sex; Outcome Measurements of Desire

\section{Introduction}

Associations Between Sexual Arousal and Desire

\asters and Johnson's human sexual 1 response cycle [1] describes sexual response as a universal, linear process consisting of four phases based on physiological changes: excitement, plateau, orgasm, and resolution. Kaplan [2,3] and Lief [4] modified the Masters and Johnson model by adding an initial desire phase, which they described as "an appetite or drive" and "a readiness for sexual activity," respectively. Although Kaplan acknowledged some of the complexities involved in the sexual response (e.g., that arousing sexual experiences could sometimes occur in the absence of desire), many interpretations of the Masters and Johnson/Kaplan model as used in research or clinical practice position desire as a drive that serves as a prerequisite to arousal, occurs independently of context, and manifests itself in the form of spontaneous sexual thoughts and urges (see critiques: $[5,6])$. The linear Masters and Johnson/Kaplan model and resulting interpretations of desire have been critiqued by researchers who question the linear model's applicability to 
real-life sexual experiences and its focus on genital responses rather than social context [6-11].

More recent models of sexual response have challenged the traditional view by arguing that desire is responsive to context rather than spontaneous [6-8]. Incentive Motivation Models applied to sexuality propose that encountering sexual stimuli triggers arousal, which then results in sexual desire $[6,8,12]$. These models emphasize that sexual stimuli may take the form of either sensory cues (e.g., from a partner or erotica) or inner thoughts, and that desire represents the conscious recognition of arousal to context $[6,8,12]$. Basson [7] applied this work to the experiences of women seeking clinical attention for sexuality-related issues in her Female Sexual Response model. According to this model, though women may sometimes choose to engage in sexual activity for sexual reasons, they also may decide to engage in sexual activity for nonsexual reasons (e.g., emotional intimacy) and feel sexual desire only after experiencing stimulation and arousal.

Though what might be called "responsive desire" (i.e., desire triggered by a stimulus or context as opposed to internal/spontaneous desire) has been theorized mainly with regard to women's sexuality $[5-8,13]$, evidence indicates that desire responds to context in men as well [14]. In a focus group study, men indicated that desire could follow rather than precede arousal, and that contextual factors (e.g., partner's desire) could enhance or inhibit their desire and arousal [15]. Men indicated considerable overlap between arousal and desire and described arousal-desire links differently for partnered and solitary activity [15]. In a comprehensive review of research on men's sexual desire, Brotto [14] emphasized gender similarities in research findings about the way desire is experienced, including the variety of contextual triggers for desire and concurrent experience of psychological arousal and desire. Thus, suggestions that desire can follow arousal seem relevant to men's sexuality, and frameworks that separate "male," automatic, and spontaneous desire from "female," responsive, and relational desire may be inaccurately essentializing and gendering desire [16].

\section{Effects of Sexual Stimuli on Sexual Desire}

Does exposure to a sexually arousing stimulus activate sexual desire? Although this is a crucial assumption embedded within the above theoreti- cal models of responsive desire, few studies have investigated this question empirically. Though laboratory studies more typically assess psychological or self-reported sexual arousal after exposure to sexual stimuli (e.g., $[17,18])$, several recent studies have also assessed participants' current feelings of sexual desire post-sexual stimuli. These studies have often focused on comparisons between clinical and nonclinical samples $[19,20]$ or hormone administration and placebo groups [21]. For example, Heiman et al. [20] reported that women with Hypoactive Sexual Desire Disorder (HSDD) had lower post-stimuli desire ratings (ratings were averaged for fantasy and erotic films with varying content) than women without HSDD, but this was only true for women not using oral contraceptives. Further studies have compared stimulus-induced desire among stimuli of the same modality but varying content, finding that sexual films depicting a coercive encounter elicited less desire than films depicting consensual encounters [22], and sexual audiotapes with anxiety- or anger-inducing dialogue elicited less desire in men than sexual audiotapes with positive dialogue [23], suggesting that desire is contextdependent.

Most studies that have assessed stimulusinduced desire have not compared multiple stimulus modalities, with a couple exceptions. Graham et al. [24] found that women reported greater desire for sex with a partner after an erotic film than after fantasy. By contrast, Toledano and Pfaus [25] found that women reported stronger motivational aspects of sexual desire and arousal after erotic fantasy than after erotic film, and men reported similar levels of desire and arousal after both stimuli. Thus, whether various stimulus modalities differentially affect desire is still an open question, given that the majority of studies have only assessed post-film desire, and the few studies that included multiple stimulus modalities have reported conflicting results.

Perhaps more importantly, these previous studies have not directly addressed the question of whether sexual stimuli activate sexual desire, because they have not compared desire in response to sexual stimuli with desire in response to nonsexual control stimuli. In one exception, Both and colleagues [26] found that viewing a sexual film increased self-reported "lust" measured post-film and the likelihood of engaging in sexual activity in the following 24 hours compared with a neutral film in women and men, but there was no effect of the sexual film on sexual desire in the 24-hour 
period. Although this study did provide an experimental test of whether sexual stimuli activate sexual desire, the researchers only examined the effects of one stimulus modality - film-on desire. Thus, it is unknown whether other non-film types of sexual stimuli (e.g., erotic stories, sexual thoughts) could evoke desire compared with nonsexual control stimuli. Given the suggestive results of Toledano and Pfaus [25] and the potential importance of fantasy in eliciting desire due to its self-determined content [27], further study on the effects of sexual fantasy conditions on desire seems especially promising.

\section{"State" and "Trait" Desire}

Increasingly, researchers have recognized that desire responds to contextual influences, and thus the concept of "state desire," or desire that can change based on the situation, has emerged $[13,20]$. Responsive desire, or desire triggered by a sexual stimulus or awareness of sexual arousal, might be considered one form of state desire $[7,8,13]$. Results from laboratory studies that assess state desire (e.g., current feelings of sexual desire) together with arousal indicate that state desire correlates with genital and psychological arousal to erotica $[21,26]$. However, desire is also commonly measured as a "trait" variable; i.e., individuals have characteristic, stable levels of desire that can be compared. Despite the fact that trait desire is conceptualized as unchanging, research suggests that even trait desire may be influenced by variables such as life events (e.g., pregnancy), relationship or partner factors, and stress [28-31]. And, research shows that the desire and arousal domains of the Female Sexual Function Index (FSFI), a "trait" measure of sexual function, are highly correlated $[32,33]$ — though multiple interpretations are possible; perhaps experiences of arousal help shape trait desire over time.

If all desire, even so-called trait desire, is context-specific, what information do people use when judging their own level of trait desire? Though commonly used questionnaire measures such as the FSFI and the Sexual Desire Inventory (SDI) instruct participants to consider their level of desire during the past month [33,34], participants' perceptions of their past-month desire could be influenced by current feelings of arousal or their evaluations of recent sexual experiences. Could exposure to sexual stimuli affect not only state desire (current feelings of desire) but perceptions of typical levels of desire? Previous research suggests that a state of sexual arousal can influence variables typically conceptualized as traits (e.g., preferences for sexual behaviors, attitudes toward condoms, propensity to engage in sexually coercive behaviors) [35]. Relevant to desire specifically, one previous study that measured trait desire with the SDI after sexually arousing conditions found no difference between film and fantasy conditions in SDI scores [25]; however, the lack of a nonarousing control condition limited interpretation of results.

In addition to trait vs. state considerations, distinctions between solitary and partnered (or dyadic) sexual desire are also relevant. Interest in behaving sexually by oneself and interest in sexual activity with a partner are empirically separate constructs that may represent distinct goals (e.g., tension release vs. intimacy) [34,36], and it is unknown whether solitary and dyadic desire respond differently to various modalities of sexual stimuli. There is evidence that these constructs, though moderately associated, show divergent associations with other parameters (e.g., solitary and dyadic desire show opposing correlations with testosterone $[17,37,38])$.

\section{Sexual Arousal in Response to Different Modalities of Sexual Stimuli}

Although very little research has addressed how desire might be differentially affected by external vs. internal stimuli, various studies have compared how fantasy vs. other modalities affect arousal. Studies that have compared effects of various sexual stimuli on arousal have shown that fantasy often leads to the lowest levels, albeit still meaningfully high arousal. The absence of sensory stimuli in fantasy is interpreted as leading to less genital and self-reported sexual arousal than other modes of stimulation, including film, photographs, written text, and spoken text $[24,39-44]$ cf. $[25,45]$. Why might fantasy produce lower arousal than other modalities in the majority of studies? One possibility involves the types of fantasy instructions used. Previous studies have employed either very broad fantasy instructions $[24,39,43]$ or very specific instructions designed to control for content between fantasy and other modalities $[41,42]$. Arguably, fantasy instructions that are too broad may not promote sufficient engagement and focus to maintain arousal, whereas instructions that are overly specific may limit participants' ability to imagine their individual preferences and thus dampen arousal. Evidence indicates that lack of engagement with the imagined situation is likely one reason for lower arousal to fantasy, as Kouk- 
ounas and Over [42] reported that men's stronger arousal to film than to fantasy was partially attributable to participants' greater absorption in the film.

Despite the fact that fantasy generally elicits less arousal than sexual stimuli such as movies or stories, fantasy has several advantages as a method for inducing arousal in research studies since it still elicits high levels of arousal. Fantasy allows individuals to tailor their mental imagery to fit their individual preferences, perhaps increasing participants' comfort with sexual material and decreasing negative affect that can be associated with erotica [46]. Using fantasy as a stimulus facilitates inclusion of sexually and ethnically diverse participants in research, since inclusion criteria do not have to be limited to a group likely to be aroused by a particular visual or textual stimulus, and researchers can bypass the problematics of using erotic material that includes only representations of majority, homogenous, or heteronormative imagery or standards. Thus, use of fantasy in research is theoretically desirable if it could elicit adequate arousal.

Recently, we introduced a new method for eliciting sexual arousal, the Imagined Social Situation Exercise (ISSE) [47]. In the ISSE, participants imagine a positive sexual encounter with another person and respond to open-ended questions about their imagined encounter. The ISSE has many of the benefits of fantasy: unlike stimuli such as films, pictures, and stories, which may require some individuals to view or read non-preferred stimuli, the ISSE allows participants to select the characteristics of a person and situation arousing to them. This is important considering that selfreported arousal is strongest in response to stimuli that reflect one's preferences [46,48]. Moreover, we have argued that the ISSE allows for more self-determination and avoids reifying negative, homogeneous, or stereotypical portrayals of sexual individuals or activities [47]. Additionally, the writing involved in the ISSE may facilitate greater absorption and thus greater arousal than fantasy alone, considering that writing about fantasy is positively linked with arousal [49], and that the open-ended ISSE questions target specific characteristics of the situation (e.g., sexual and emotional responses, personal involvement in the situation) linked with arousal $[48,50]$. Though our previous work showed that the ISSE increased psychological sexual arousal relative to nonsexual control conditions (positive, stressful, and neutral imagined situations) [47], it is unknown how the ISSE will compare with other sexual conditions in terms of arousal. This comparison between the ISSE and other sexual modalities is critical considering that fantasy is often found less arousing than external stimuli but the ISSE is distinguishable from "plain" fantasy conditions used in previous research.

\section{Aims}

In the current study, we examined and compared (i) effects of four conditions on self-reported sexual arousal: the ISSE, unstructured fantasy (in which participants imagine sexually arousing situations but do not write about them), an erotic story, and a neutral condition (describing places); (ii) how exposure to sexually arousing stimuli affects state and so-called trait sexual desire; and (iii) whether levels of arousal and desire are associated. We also explored whether the above effects were moderated by gender, given the relevance of responsive desire to men's sexuality as well as women's [14], and the potential for modalityinduced arousal to be gender-specific [25,51]. Participants completed baseline arousal and state desire scales and their randomly assigned condition (ISSE, unstructured fantasy, erotic story, or neutral), followed by a post-activity arousal scale, state desire scale, and the SDI [34], which was used to measure trait desire. We hypothesized that all three sexual conditions would increase arousal compared with the neutral condition, and explored whether the ISSE would elicit comparable arousal to the more standard unstructured fantasy and erotic story. With respect to sexual desire, we predicted that participants would report greater state and trait desire after sexual conditions compared with the neutral condition; i.e., that exposure to sexually arousing stimuli would activate desire. Finally, we hypothesized that higher levels of sexual arousal in response to sexual stimuli would predict higher levels of sexual desire.

\section{Methods}

\section{Participants}

Participants were recruited for an online study through posters, online advertisements, and Amazon's MTurk, which has been successfully used in previous psychology research [52]. Participants completed an online screening to ensure that they were over 18, living in the United States, comfortable with erotica, and in a private place. The final 
sample, which included participants who qualified for the study and completed enough of the survey to be used in analyses, consisted of 223 participants (128 women, $\left.95 \mathrm{men}^{1}\right)$. The mean age of participants was 28 years (standard deviation $[\mathrm{SD}]=9$ years). About half $(\mathrm{N}=101)$ of the participants were students (46 of whom were also employed), 96 participants were employed nonstudents, and 24 participants were neither students nor employed. Participants self-identified their race/ ethnicity, and we categorized such that there were 11 African American/black, 18 Asian, 8 bi-/ multiracial, 10 Hispanic/Latina(o), 2 Middle Eastern, 2 Native American, and 170 white participants. Most participants $(\mathrm{N}=209)$ had some college or other post-high-school training, and some $(\mathrm{N}=50)$ had children. Participants selfidentified their sexual orientation; 165 identified as heterosexual, 18 as bisexual, 6 as homosexual, 5 as lesbian, 3 as bi-curious, and one each as gay, pansexual, queer, queer heterosexual, same gender loving, and with a same-sex partner. Participants indicated their relationship status based on definitions we provided [53]; 136 participants were in committed relationships, 44 were in casual relationships, and 39 were single. Participants reported using erotica three to four times per month on average and engaging in elaborate sexual fantasy an average of once per week. There were no significant differences in the above variables by gender, with the exceptions that men were more likely than women to be employed $(P=0.046)$, and men reported more frequent use of erotica $(P<0.001)$ and sexual fantasy $(P<0.001)$ than women.

\section{Materials}

\section{Health and Background Questionnaire}

This questionnaire included items used to describe the sample, such as age, occupation status, and race/ethnicity.

\section{Affect and Arousal Scale [54]}

This scale is commonly used to measure state affect and arousal in response to erotic stimuli (e.g., [17]), and we have used it successfully in the past post-ISSE [47]. The scale included 34 items which made up six a priori subscales [17]: perceived genital sexual arousal (e.g., "genital pulsing

\footnotetext{
${ }^{1}$ Though we had one agendered and one genderqueer individual participate, only women and men were included in analyses due to the use of gender as a moderating variable and the small sample sizes for genders other than female or male.
}

or throbbing"), psychological sexual arousal (e.g., "sensuous"), perceived autonomic arousal (e.g., "perspiration"), positive affect (e.g., "interested"), negative affect (e.g., "embarrassed"), and anxiety (e.g., "worried"). Internal consistency was high for each of the subscales, with Cronbach's alphas ranging from 0.74 to 0.94 . Women and men were provided with identical versions of the scale with the exceptions that only men were asked about erections, and men were asked about nipple sensations while women were asked about breast sensations. Participants were asked to rate how well each word or phrase described their current feelings on a scale from " 1 " = "Not at all" to "7" = "Intensely."

\section{State Desire Items}

State sexual desire was measured with two items: "a desire to masturbate" (solitary state desire) and "a desire for sexual activity with a partner" (dyadic state desire) [24]. Participants rated the extent to which each item described their current feelings on a scale from " $1 "=$ "Not at all" to "7" = "Intensely."

\section{ISSE [47]}

In the ISSE, participants imagined and described a positive sexual encounter with another person. Participants read a prompt instructing them to imagine that they were engaging in enjoyable sexual activity with an attractive partner. Then, they responded to open-ended questions asking them to describe the attractive person, their feelings of attraction, the situation and background events, and the sexual activity. Past studies have shown that the ISSE increases self-reported sexual arousal compared with positive and neutral control conditions in women and men [47,55]. The prompt for the ISSE read as follows, and see ref. 47 for the full text of the ISSE including the openended questions.

$$
\begin{aligned}
& \text { Imagine that you have met a person who is sexually } \\
& \text { attractive to you, and you and this person decide to } \\
& \text { engage in sexual activity together. You find your sexual } \\
& \text { partner very attractive, and you enjoy the sexual activity } \\
& \text { very much. Consider your sexual partner, your feelings } \\
& \text { of attraction, how you start being sexual, and the sexual } \\
& \text { activity itself. }
\end{aligned}
$$

\section{Unstructured Fantasy}

In the unstructured fantasy condition, participants were asked to engage in sexual fantasy for 10 minutes. The 10-minute time period was chosen for consistency with the average length of time spent on the ISSE in previous studies [47,55], and the survey program timed the 10 minutes and 
advanced when the time had completed. The instructions for this condition were as follows, and were adapted from previous studies [24,25]:

\begin{abstract}
For the next 10 minutes, please fantasize about a sexual experience or situation that would lead you to feel sexually aroused. You may fantasize about anything you wish; we will not ask you about the content of your fantasies. You may keep your eyes open or closed, whichever is more comfortable for you.
\end{abstract}

\section{Erotic Story}

The erotic story used in this study was an excerpt from the romance novel Set the Dark on Fire [56]. The excerpt described a sexual encounter between a woman and a man that included foreplay, cunnilingus, and intercourse, from a third person point of view. The encounter was portrayed as arousing and pleasurable for both characters. Pilot testing indicated that the story took about 8-10 minutes to read and was arousing.

\section{Neutral Condition}

In the neutral condition, participants were asked to describe the room they were currently located in, and another location they had been that day, in as much detail as possible. The location descriptions were intended to keep participants engaged in mental imagery and writing about an emotionally neutral topic, and accordingly control for many of the features of the ISSE. Participants described the current room for 5 minutes and another location for 5 minutes, resulting in 10 minutes total spent on the neutral condition.

\section{SDI [34]}

The SDI is a measure of sexual desire in which participants indicate the strength, frequency, and importance of fulfilling their solitary and partnered sexual desire. Several items specify a reference period of the past month, so the SDI is considered a "trait" measure of desire [57,58], which was appropriate given that we were specifically interested in how even perceptions of typical levels of desire might be influenced by sexual stimuli. We modified the SDI by adding one question: "During the last month, how often have you had sexual thoughts?" [59]. The SDI includes 15 questions, most of which are answered on an eight-point scale (e.g., "1" = "No desire"; " 8 " = "Strong desire"). The inventory results in a solitary subscore, a dyadic (partnered) subscore, and a total score (which includes the solitary and dyadic items as well as a few general items). The SDI is a valid and appropriate measure of sexual desire in nonclinical populations of varied ages and sexual experiences $[17,34,37,60-62]$. Internal con- sistency of the SDI was high in our sample (Cronbach's alpha $=0.83$ for solitary SDI, 0.83 for dyadic SDI, and 0.86 for total SDI).

\section{Relationships and Sexuality}

These questions characterized participants in terms of their relationship status, sexual experience, use of erotica (on a scale from " 0 " = "Not at all" to " $6 "=$ "More than once/day"), and frequency of sexual fantasy.

\section{Procedure}

The entirety of the study was conducted online, enabling subjects to participate in a comfortable, ecologically valid non-laboratory environment. Participants first completed a screening questionnaire and an informed consent form. All participants completed the health and background questionnaire, a baseline Affect and Arousal Scale, and baseline state desire items. Then, participants proceeded to their randomly assigned condition: ISSE, unstructured fantasy, erotic story, or neutral. Immediately following the condition, participants completed a post-activity Affect and Arousal Scale and the state desire items, followed by the SDI. Finally, participants responded to the relationships and sexuality questionnaire. As compensation for their time, participants received either $\$ 0.50$ (if they participated through Amazon's MTurk) or the chance to enter a raffle for one of two $\$ 50$ prizes (if they participated via regular online recruitment).

\section{Analyses}

We analyzed our data with SPSS 18.0 (SPSS Inc., Chicago, IL, USA). To test for effects of condition on arousal and state desire, we used repeated measures analyses of covariance (RM ANCOVAs) with multiple measures. Following significant interactions, we tested for simple main effects with to multivariate analyses of covariance (MANCOVAs) and least significant difference (LSD) post hoc tests, as the omnibus MANCOVA holds the family-wise error rate constant. To examine effects of condition on trait desire, we used a MANCOVA and followed up significant effects with LSD post hoc tests. We controlled for age, relationship status, and frequency of erotica use, given that these variables have been linked with desire and/or arousal $[17,63,64]$, as well as sexual identity, since preferred stimuli elicit greater psychological arousal than non-preferred stimuli, and our stimulus conditions differed in their flexibility to match individual preferences [18,65]. Including these control variables as covariates did meaningfully 
alter the pattern of results, so we report results with the covariates included in the model. For purposes of analyses, relationship status was categorized as single, casually partnered, or committed; and sexual identity was categorized as heterosexual, bisexual or lesbian/gay/queer based on self-identification. Categorical variables were contrast-coded when used as covariates. We used $P<0.05$ as the criterion for significance and we reported trends at $P<0.10$. Sample sizes vary between analyses due to missing data.

\section{Main Outcome Measures}

The main outcome measures in this study were perceived genital arousal, psychological arousal, perceived autonomic arousal, anxiety, positive affect, and negative affect as measured by the Affect and Arousal Scale [54] pre- and postcondition; solitary and dyadic state desire as measured by two items [24]; and solitary and dyadic trait desire as measured by the SDI [34].

\section{Results}

\section{Effects of Condition on Arousal and Affect}

To examine effects of the conditions on affective responses, we conducted an RM ANCOVA with time as the within-subjects independent variable, condition and gender as between-subjects independent variables, control variables (age, relationship status, erotica use, and sexual identity) as covariates, and the six Affect and Arousal subscales as dependent variables. There was a significant multivariate main effect of gender, $F(6$, $182)=2.58, P=0.020$, such that across time and conditions, women reported higher perceived genital arousal than men, $F(1,187)=6.52$, $P=0.011$. There was also a significant multivariate interaction between time and condition for affect and arousal, $F(18,515)=6.08, P<0.001$, with significant univariate interactions (further explained below) between time and condition for all subscales (all $P$ 's $<0.05$ ) except anxiety (not significant). We then proceeded with post hoc tests to follow up these significant interactions between time and condition.

There were no significant differences in affect and arousal between conditions at Time 1, all $P$ 's $>0.10$. At Time 2, all three sexual conditions elicited higher perceived genital arousal, psychological arousal, perceived autonomic arousal, and positive affect compared with the neutral condi- tion, all $P$ 's $<0.001$. The sexual conditions did not differ strongly from each other, however. There were trends for higher perceived genital arousal after fantasy than the story $(P=0.067)$ and ISSE $(P=0.063)$, as well as for higher psychological arousal after fantasy than the story $(P=0.066)$. There were also trends for higher perceived autonomic arousal after fantasy than the story $(P=0.073)$ and ISSE $(P=0.095)$. There were no significant differences between the three sexual conditions in positive affect, and no significant differences between the four conditions in negative affect at Time 2, all $P$ 's $>0.10$. Thus, all three sexual conditions increased arousal and positive affect compared with the neutral condition, with a tendency for fantasy to be most arousing.

In terms of changes from Time 1 to Time 2, arousal (perceived genital, psychological, and perceived autonomic) increased significantly in all three sexual conditions from Time 1 to Time 2 as expected, all $P$ 's $<0.001$. In the neutral condition, psychological arousal decreased significantly from Time 1 to Time $2(P=0.003)$, and perceived genital and perceived autonomic arousal were unchanged $(P$ 's $>0.10)$. Positive affect increased significantly from Time 1 to Time 2 in all three sexual conditions (all $P$ 's $<0.001)$ and decreased significantly in the neutral condition $(P<0.001)$. Finally, negative affect increased significantly from Time $1 \quad($ mean $=1.32, \mathrm{SD}=0.40)$ to Time 2 $($ mean $=1.51, \mathrm{SD}=0.69)$ in the ISSE condition $(P=0.035)$ but remained unchanged in the other conditions (all $P$ 's $>0.10$ ). Therefore, all sexual conditions increased sexual arousal and positive affect, and the ISSE additionally increased negative affect, from baseline to post-activity. See Figure 1 for means and standard errors of Affect and Arousal subscales at Time 1 and Time 2.

\section{Effects of Condition on State Desire}

To examine effects of condition on state desire, we conducted an RM ANCOVA with time as the within-subjects independent variable, condition and gender as between-subjects independent variables, control variables (age, relationship status, erotica use, and sexual identity) as covariates, and solitary and dyadic state desire as dependent variables. There was a significant multivariate effect of time, $F(2,185)=5.91, P=0.003$, such that both solitary and dyadic state desire increased from Time 1 to Time 2, P's $<0.001$. Furthermore, there was a significant multivariate interaction between time and condition, $F(6,370)=11.13, P<0.001$, with significant univariate interactions for solitary 


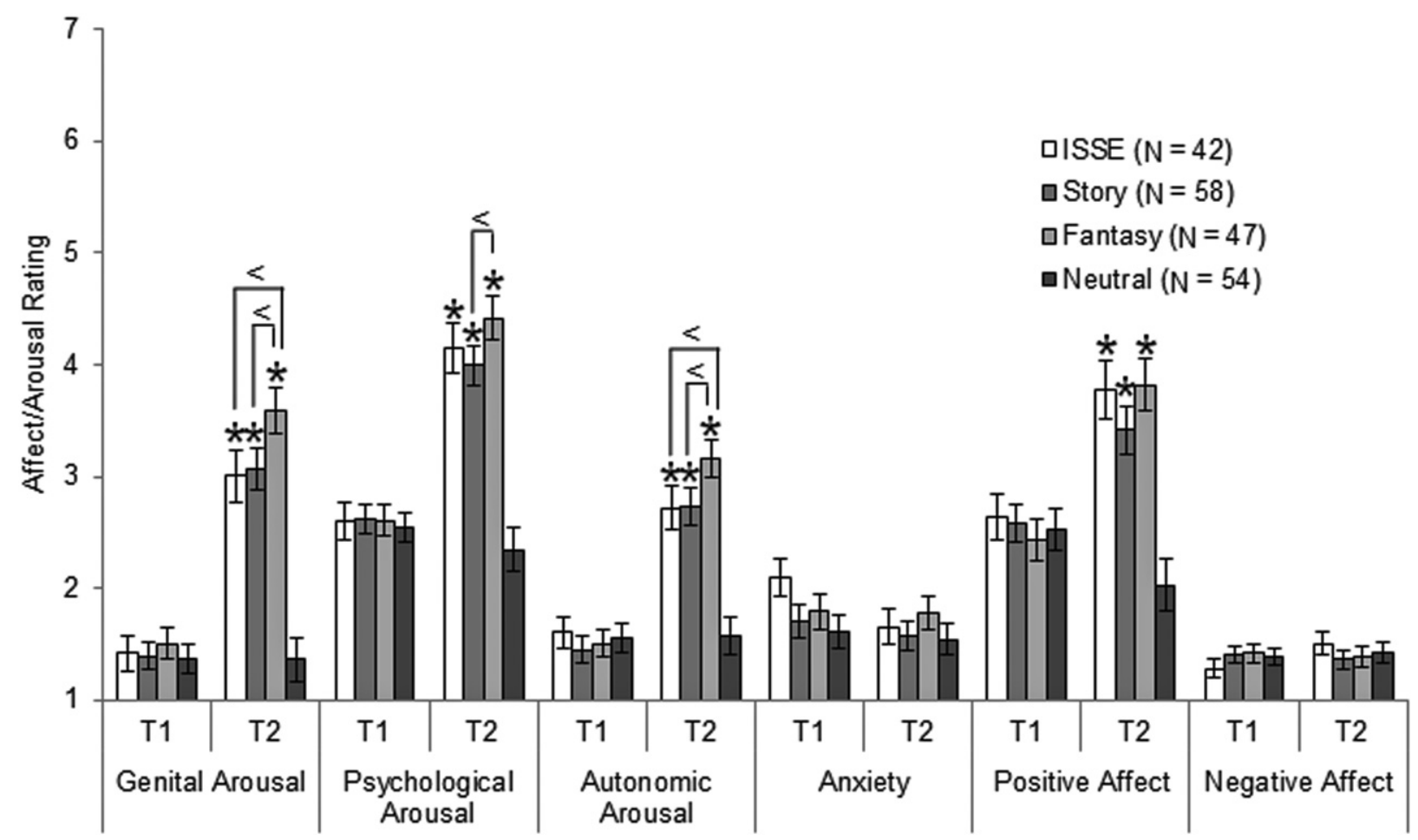

Figure 1 Affect and Arousal at Time 1 and Time 2 for the Imagined Social Situation Exercise (ISSE), Story, Fantasy, and Neutral conditions. Participants responded on a scale from "1" = "Not at all" to "7" = "Intensely." "*” indicates a significant difference from the Neutral condition at Time 2 at $P<0.05$, and " $<$ " indicates a trend at $P<0.10$. Note: No significant differences between conditions at Time 1.

state desire, $F(3,186)=15.99, P<0.001$, and dyadic state desire, $F(3,186)=21.44, P<0.001$. There were no significant differences between conditions in solitary or dyadic state desire at Time 1, P's $>0.10$. At Time 2, solitary and dyadic state desire were significantly higher in all three sexual conditions than the neutral condition, all $P$ 's $<0.01$. There were no significant differences between the three sexual conditions in state desire, all $P$ 's $>0.10$. Thus, sexual conditions increased solitary and dyadic state desire compared with the neutral condition (see Figure 2).

\section{Effects of Condition on Trait Desire}

We tested effects of condition on trait sexual desire using a MANCOVA with condition and gender as

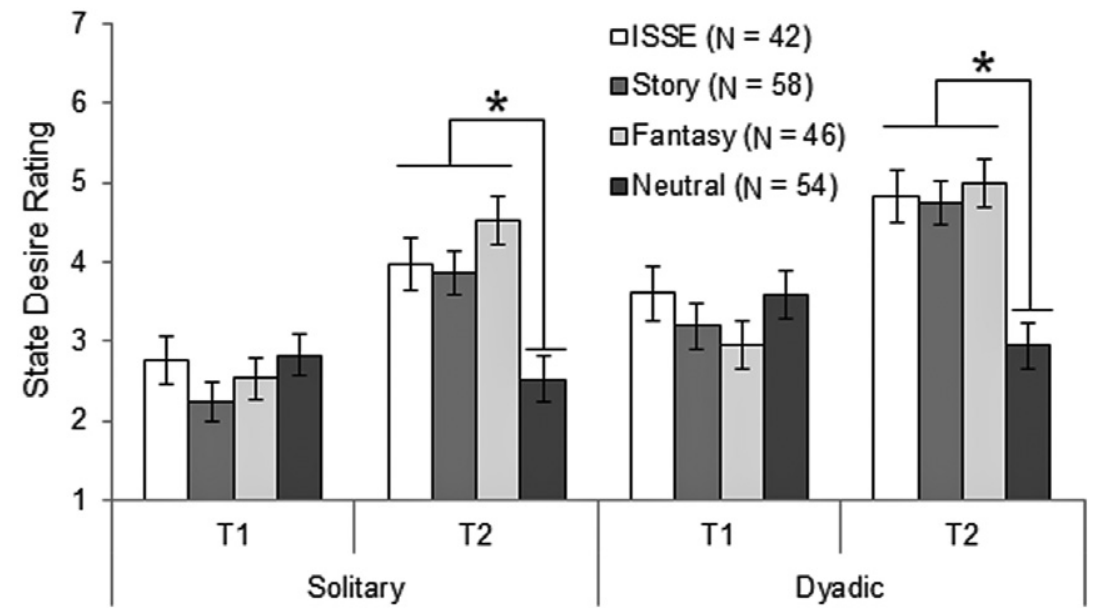

Figure 2 Solitary and Dyadic State Desire at Time 1 and Time 2 for the Imagined Social Situation Exercise (ISSE), Story, Fantasy, and Neutral conditions. Participants responded on a scale from " $1 "=$ "Not at all" to "7" = "Intensely." "*”" indicates a significant difference at $P<0.05$. Note: No significant differences between conditions at Time 1. 


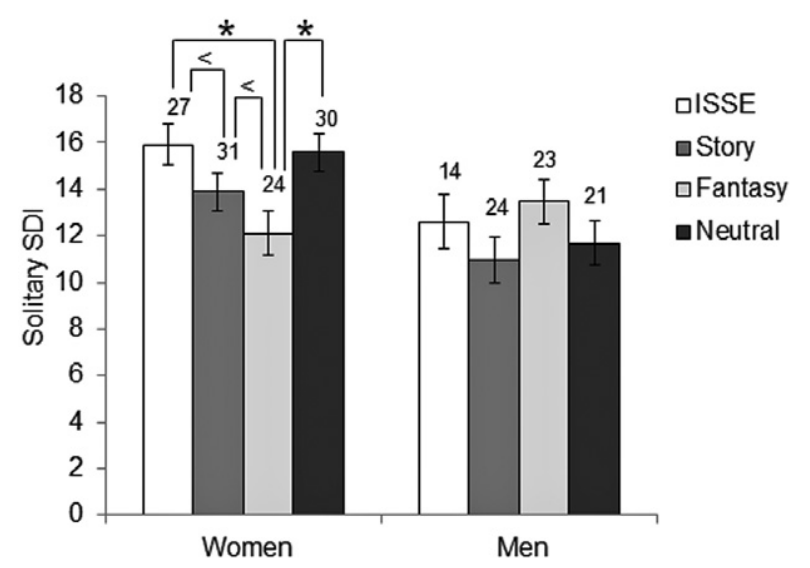

Figure 3 Solitary Sexual Desire Inventory (SDI) scores for women and men after the Imagined Social Situation Exercise (ISSE), Story, Fantasy, and Neutral conditions. Numbers at the end of each bar indicate N's for each group. "*” indicates a significant difference at $P<0.05$, and " $<$ " indicates a trend at $P<0.10$.

independent variables, control variables (age, relationship status, erotica use, and sexual identity) as covariates, and solitary, dyadic, and total SDI scores as the dependent variables. There was a significant multivariate interaction between condition and gender, $F(3,180)=3.84, P=0.011$, and a significant condition by gender interaction for solitary SDI, $F(3,180)=3.39, P=0.019$. We thus examined post hoc differences separately by gender.

In women, solitary SDI was significantly higher after the ISSE $(P=0.001)$ and neutral $(P=0.003)$ conditions compared with fantasy, with a trend for higher solitary SDI after the ISSE than the story $(P=0.052)$. There was also a trend for women's solitary SDI to be higher after the story than fantasy $(P=0.099)$. In men, there were no significant differences between conditions in solitary SDI, all $P$ 's $>0.10$. Thus, women's trait solitary sexual desire was highest after the ISSE and neutral conditions and lowest after fantasy, but men's trait desire did not respond to conditions (see Figure 3).

There was no significant main effect of condition on dyadic or total SDI scores, and no significant condition by gender interaction for either dyadic or total SDI, all P's $>0.10$. Therefore, though condition affected solitary SDI, it did not influence dyadic or total SDI.

\section{Associations Between Arousal and Desire}

Because desire variables differed by condition, we were interested in whether levels of arousal or positive affect in response to sexual stimuli would predict levels of desire for participants in the three sexual conditions (ISSE, unstructured fantasy, and story). To determine whether arousal and positive affect predicted levels of sexual desire, we conducted multiple linear regression analyses with arousal, positive affect, gender, and control variables (age, relationship status, sexual identity, and erotica use) as predictors and desire variables as outcome variables. We focused on solitary state desire, dyadic state desire, and solitary SDI as our outcome variables, as these desire variables differed based on stimulus condition. For arousal, positive affect, and state desire, we used change scores (Time 2-Time 1) in these analyses. Because genital, psychological, and autonomic arousal were strongly intercorrelated ( $r$ values of 0.65 and above), we created a composite arousal measure by averaging the changes in genital, psychological, and autonomic arousal to avoid issues of multicollinearity.

For solitary state desire, the model explained $42.5 \%$ of the variance $\left(\mathrm{R}^{2}\right.$ adj $\left.=0.387\right), F(9$, $136)=11.16, P<0.001$. The arousal composite significantly positively predicted solitary state desire, $\beta=1.07, t(136)=7.03, P<0.001$. Positive affect was not a significant predictor of solitary state desire, $\beta=-0.08, t(136)=-0.69, P=0.493$ (see Table 1a).

The predictors accounted for $31.9 \%$ of the variance in dyadic state desire $\left(\mathrm{R}^{2}\right.$ adj $\left.=0.274\right), F(9$, $137)=7.12, \quad P<0.001$. Similar to above, the arousal composite significantly positively predicted dyadic state desire, $\beta=0.72, t(137)=4.61$, $P<0.001$, but positive affect was not a significant predictor, $\beta=0.09, t(137)=0.74, P=0.460$ (see Table $1 b)$.

Finally, the model explained $36.7 \%$ of the variance in solitary SDI $\left(\mathrm{R}_{\text {adj }}^{2}=0.325\right), \quad F(9$, $137)=8.82, P<0.001$. However, the arousal composite was not a significant predictor of solitary SDI, $\beta=0.51, t(137)=1.20, P=0.234$, though there was a trend for higher positive affect to predict higher solitary SDI, $\beta=0.59$, $t(137)=1.73, P=0.085$ (see Table $1 \mathrm{c}){ }^{2,3}$

Including the two-way arousal*gender, positive affect ${ }^{*}$ gender, and arousal*positive affect interactions or the three-way arousal*positive affect*gender interaction did not significantly increase the percentage of variance explained in any desire variables.

${ }^{3}$ Though Time 1 variables (Time 1 state desire, arousal, and positive affect) were significant predictors in some models, including Time 1 variables as predictors did not alter the main pattern of results. 
Table 1 Results of a multiple regression predicting (a) solitary state desire, (b) dyadic state desire, (c) solitary Sexual Desire Inventory from an arousal composite, positive affect, and control variables*

\begin{tabular}{|c|c|c|c|}
\hline & $\beta$ (SE) & $t$ value & $P$ value \\
\hline \multicolumn{4}{|l|}{ a } \\
\hline Arousal composite & $1.07(0.15)$ & 7.03 & $<0.001$ \\
\hline Positive affect & $-0.08(0.12)$ & -0.69 & 0.493 \\
\hline Gender $^{\dagger}$ & $-0.42(0.16)$ & -2.59 & 0.011 \\
\hline Age & $-0.02(0.02)$ & -1.37 & 0.173 \\
\hline Relationship status $1^{\ddagger}$ & $0.31(0.21)$ & 1.50 & 0.136 \\
\hline Relationship status $2^{\ddagger}$ & $-0.17(0.09)$ & -1.90 & 0.059 \\
\hline Sexual identity $1^{\ddagger}$ & $-0.24(0.20)$ & -1.20 & 0.233 \\
\hline Sexual identity $2^{\ddagger}$ & $-0.15(0.15)$ & -0.99 & 0.322 \\
\hline Erotica use & $-0.24(0.10)$ & -2.57 & 0.011 \\
\hline \multicolumn{4}{|l|}{$b$} \\
\hline Arousal composite & $0.72(0.16)$ & 4.61 & $<0.001$ \\
\hline Positive affect & $0.09(0.12)$ & 0.74 & 0.460 \\
\hline Gender $^{\dagger}$ & $-0.07(0.16)$ & -0.43 & 0.671 \\
\hline Age & $-0.02(0.02)$ & -1.01 & 0.314 \\
\hline Relationship status $1^{\ddagger}$ & $0.24(0.21)$ & 1.11 & 0.269 \\
\hline Relationship status $2^{\ddagger}$ & $-0.18(0.09)$ & -1.97 & 0.051 \\
\hline Sexual identity $1^{\ddagger}$ & $-0.30(0.21)$ & -1.46 & 0.148 \\
\hline Sexual identity $2^{\ddagger}$ & $0.13(0.16)$ & 0.82 & 0.416 \\
\hline Erotica use & $-0.12(0.10)$ & -1.29 & 0.200 \\
\hline \multicolumn{4}{|l|}{ c } \\
\hline Arousal composite & $0.51(0.43)$ & 1.20 & 0.234 \\
\hline Positive affect & $0.59(0.34)$ & 1.73 & 0.085 \\
\hline Gender $^{\dagger}$ & $0.81(0.45)$ & 1.80 & 0.074 \\
\hline Age & $0.08(0.05)$ & 1.70 & 0.091 \\
\hline Relationship status $1^{\ddagger}$ & $-0.62(0.59)$ & -1.05 & 0.295 \\
\hline Relationship status $2^{\ddagger}$ & $0.28(0.25)$ & 1.10 & 0.272 \\
\hline Sexual identity $1^{\ddagger}$ & $-0.58(0.58)$ & -1.00 & 0.318 \\
\hline Sexual identity $2^{\ddagger}$ & $-0.16(0.43)$ & -0.37 & 0.710 \\
\hline Erotica use & $1.64(0.27)$ & 6.16 & $<0.001$ \\
\hline
\end{tabular}

*Analysis included only participants in the sexual (Imagined Social Situation Exercise, unstructured fantasy, and story) conditions. All continuous variables were centered prior to analysis

${ }^{\dagger}$ Coded as $1=$ female, $-1=$ male

TThe categorical variables of relationship status and sexual identity were each represented as two contrast codes in the regression

$\mathrm{SE}=$ standard error

\section{Instruction Compliance Check}

At the end of the survey, participants were asked to report any activities they were currently engaging in (only this survey, chatting with friends, etc.) as a check to determine the extent to which participants were focused on the research study vs. other activities. Out of the 223 participants, 12 did not respond to this item, leaving 211 participants for this analysis. The majority of participants $(\mathrm{N}=168)$ reported that they were only completing the survey, or completing the survey with music or other background noise (e.g., TV) on. The remainder of participants reported engaging in activities such as chatting with a friend or surfing the Internet in addition to the survey. Two men in the fantasy condition reported masturbating, and one man in the fantasy condition reported viewing erotica, but excluding these three participants did not change the pattern of results.

\section{Discussion}

In the current study, we compared levels of sexual arousal and desire in response to a neutral condition and three sexual conditions: ISSE, unstructured fantasy, and erotic story. As expected, all three sexual conditions increased self-reported sexual arousal compared with the neutral condition. Unstructured fantasy elicited the largest increases in arousal, though differences between fantasy and the other sexual conditions were relatively small. We also investigated how sexual stimuli affected state and trait sexual desire. As predicted, sexual stimuli increased solitary and dyadic state desire compared with neutral stimuli. In addition, our results demonstrated that perceptions of typical solitary sexual desire responded to context in women, such that women had the highest solitary SDI scores after the ISSE and neutral conditions and lowest solitary SDI after fantasy. However, trait desire did not respond to context in men. Larger increases in arousal predicted larger increases in state desire, suggesting that effects of sexual stimuli on state desire were at least partially due to increases in arousal.

\section{Effects of the ISSE vs. Other Sexual Conditions on Arousal}

Our results support the use of the ISSE in sexuality research, as this condition like the other sexual conditions increased perceived genital arousal, psychological arousal, and perceived autonomic arousal relative to the neutral condition. The ISSE elicited a similar level of arousal to the story condition, which means that the ISSE elicited comparable arousal to a more widely used stimulus (e.g., [41,66-68]) while maintaining several advantages over a story, such as the participants' ability to self-determine the characteristics of the people, setting, and sexual behaviors described. However, our findings also highlight some trade-offs of using the ISSE. Although the ISSE produced meaningful sexual arousal as evidenced by higher arousal than the neutral condition, the degree of arousal was less than that of unstructured fantasy-albeit only at a trend level. Though we initially predicted that writing about sexual situations would maximize arousal through greater engagement, our findings suggest that arousal may be greatest when participants can fantasize in a less structured manner and/or are not inhibited by the instruction to write about the sexual situation. Overall, researchers might consider trade-offs between unstructured fantasy and ISSE when deciding which method to use: unstructured 
fantasy frees participants from the constraint of writing, but the ISSE provides data on what participants actually imagined so that these themes can be analyzed or controlled for, which might be important to research design.

Why might fantasy have elicited the most arousal in our study, in contrast to lower (albeit still significant) arousal relative to other modalities in previous studies? [24,39-44] One possibility is that unlike previous studies, our study occurred at home rather than in a laboratory environment, so fantasy conditions may be most arousing when participants are comfortable and familiar with their environment. Importantly, most previous studies did not directly compare fantasy with written text, focusing on comparisons between fantasy and film or audio [24,39,40,42-44]. Two previous studies that compared written text with structured fantasy instructions intended to match the fantasy to other stimuli reported conflicting results $[41,45]$, so unstructured but not structured fantasy may increase arousal compared with written text. Thus, type of fantasy instructions and the setting (home or lab) may be important factors when comparing fantasy with other modalities.

We found that the ISSE elicited similar levels of positive affect compared with the other sexual conditions. However, the ISSE also resulted in a small but significant increase in negative affect from Time 1 to Time 2, whereas negative affect remained unchanged in the other conditions. Given that we have argued that the ISSE allows for greater self-determination than other types of sexual stimuli [47], this result is somewhat surprising. One possibility is that while participants likely found the ISSE enjoyable (as evidenced by an increase in positive affect compared with the neutral condition as well as positive comments left at the end of the survey), the unfamiliarity of the ISSE may have led to a mixture of positive and negative affect (as participants had likely used erotica and fantasized sexually prior to the study, but were encountering the ISSE for the first time). Future research could examine whether repeated exposure to the ISSE might change affective responses to this method.

\section{Sexual Stimuli Increase Sexual Desire}

Our second research question examined whether sexually arousing stimuli would increase state desire and perceptions of typical desire; we predicted that the sexual conditions would increase desire compared with the neutral condition. This hypothesis was supported with respect to state desire; both solitary and dyadic state desire increased after the three sexual conditions compared with the neutral condition in women and men. There were no significant differences among the three sexual conditions in state desire, suggesting that state desire responds to sexual stimuli regardless of stimulus modality. Our results provide evidence supporting a central component of Incentive Motivation Models and the Basson model of sexual response-that sexual stimuli activate sexual desire. Furthermore, our evidence that state desire responds to sexual context in women and men builds on previous research suggesting that men's sexual responses, in addition to women's, may deviate from a traditional linear model $[14,15]$.

Not only do our results show that state desire responds to sexual stimuli, but they also demonstrate the fluidity of "trait" desire. We found that women perceived their typical levels of desire differently based on current engagement with sexual stimuli, as they reported higher solitary sexual desire as measured by the SDI after the ISSE relative to fantasy and story, as well as higher solitary SDI after the neutral and story conditions compared with fantasy. Higher desire after the neutral condition was surprising, but similar to a previous finding in which men showed greater approach tendencies to sexual stimuli (measured by reflex amplitude) after a neutral condition than a sexually arousing condition [26]. In our study, women likely expected a sexual stimulus since they were informed about the sexual nature of the study, and upon receiving a neutral stimulus instead, perceptions of typical desire may have increased due to the unfulfilled expectation for sexual arousal [26]. Consistent with this interpretation, women in the fantasy condition-the most arousing sexual condition-had the lowest solitary SDI scores. Our results show that sexual stimuli may influence state desire and perceptions of typical desire differently in women: exposure to sexual stimuli increases state desire, but may also lead to lower reports of typical desire. The lower trait desire may occur because sexual stimuli and desire alone-rather than sexual activity-may themselves be a goal of desire [16]; thus, desire could be fulfilled, in addition to sparked, by sexually arousing stimuli. However, this interpretation is complicated by the result that the ISSE, which was arousing, elicited reports of high trait desire. Regardless, responsive desire might thus occur in multiple ways: arousing stimuli can trigger desire, but lack of expected arousal can also enhance desire 
(though this might only apply to a nonclinical sample such as ours, and not to women with arousal disorders who consistently lack arousal).

We found effects of condition on SDI scores even though the SDI measures desire as a "trait" variable, i.e., over the past month, indicating that "trait" desire is judged differently after exposure to sexual vs. neutral stimuli. The results of the current study do not clarify whether desire itself is unstable (i.e., trait levels of desire do not really exist), or whether the measurement of desire is unstable (i.e., trait levels of desire may exist, but the measurement of trait desire may be unreliable due to arousal or other states). However, our findings do reveal that individuals' assessments of their typical desire are influenced by their engagement with sexual stimuli at the current moment, questioning whether desire can be accurately conceptualized as a long-term trait when its measurement is subject to state-like fluctuations.

Similarly, future research is needed to determine how engagement with sexual stimuli changes SDI scores; for example, might current arousal affect one's memory for past-month desire, or is assessment of past-month desire altered by comparisons to current feelings of desire (e.g., if current desire is high, do typical levels of desire seem lower)? Regardless of mechanism, these findings have implications for how desire is measured in research studies and in clinical settings, such that expectations and the context in which desire is assessed might affect its measurement. For example, if desire is measured in a clinical vs. nonclinical sample after exposure to erotica, lower desire in the clinical sample may reflect less expectation of arousal to the stimuli, rather than lower "trait" desire. This feedback between arousal and desire is relevant to current debates over Diagnostic and Statistical Manual (DSM) definitions of arousal and desire disorders, providing further evidence that definitions should address the responsive rather than spontaneous nature of desire in order to avoid over-pathologizing [5,14]. Moreover, our results suggest that context of measurement is an important issue to consider when developing and validating measures of sexual desire, in addition to factors such as reliability and content validity [69,70].

Interestingly, experimental condition affected measurement of solitary but not dyadic trait sexual desire in women, suggesting that individuals' reports of solitary desire may be more responsive to stimuli such as erotica and fantasy, while reports of dyadic desire may instead reflect the current availability or willingness of a partner. Our results-which show that state and perceptions of typical solitary desire respond to sexual context-point to the need to broaden discussions of responsive desire to include solitary desire, since most models implicitly or explicitly focus on desire for partnered sexual activity $[7,71]$. In general, masturbation, solitary sexuality, and solitary desire have been understudied in sexuality work, despite their importance in sexual pleasure and health $[13,37,62]$. Furthermore, our findings show that desire can respond to self-generated stimuli (e.g., the imagined content in the ISSE) and erotica, and the potential for desire to respond to stimuli besides partner cues is often neglected, especially in discussions of women's desire [16]. Recognizing that desire is responsive to context in women and men, and that even solitary desire is contextually situated, is important for basic and clinical understandings of arousal and desire.

Why might sexual stimuli not have influenced SDI scores in men? Although we found that sexual stimuli increased men's state desire, reports of trait desire were unaffected by sexual stimuli in men. Our results suggest that men's desire may involve some "state" components which are subject to fluctuation based on current levels of arousal, but also some trait-like components which remain relatively stable despite the sexual context. This result is compatible with a larger body of sexuality research showing greater fluidity in women's sexuality than men's in response to state-like cues (e.g., $[18,53])$. Compared with empirical research on women's desire, the nature of men's sexual desire is understudied [14], and further research is needed to clarify in what contexts men's desire can respond to arousal.

Our findings demonstrate that larger increases in sexual arousal predicted larger increases in state desire. These findings are in line with qualitative $[15,72,73]$ and quantitative $[32,74,75]$ research in women and men showing overlap in experiences of arousal and desire. Furthermore, the interrelations between arousal and desire in our data suggest that effects of sexual stimuli on state desire may be a result of sexual arousal; i.e., that sexual arousal may have increased sexual desire. However, multiple interpretations are possible: individuals who generally experience greater arousal might also generally experience greater desire, and our data do not clarify directionality or causality of these arousaldesire links. Additionally, arousal did not significantly predict solitary SDI scores, and there was a trend for positive affect to predict solitary SDI, suggesting that responses besides arousal (e.g., 
recognition of a stimulus as sexual, nonsexual affect: see [22]) may contribute to effects of sexual stimuli on measurement of trait desire, though arousal is likely one key factor in predicting state desire.

\section{Limitations and Future Directions}

One limitation of our study is that we only compared select modalities of sexual stimuli: ISSE, story, and unstructured fantasy. As film generally elicits higher arousal than other modalities [24,39,41-44], future comparisons including a film condition may be useful. Including a spoken text condition, in which participants could close their eyes while listening to erotic stimuli, might help clarify whether trends for lower arousal to the story and ISSE than fantasy might be due to the fact that participants were required to keep their eyes open in order to read or type during the story and ISSE conditions.

A further limitation of our study, as with most sexuality research, is that our sample represents a select group of individuals willing to participate in a study involving sexual content, though our use of an online study likely allowed for a broader sample than a laboratory study. Drawbacks of the nonlaboratory environment include less control over whether participants follow instructions and less ability to limit distractions; however, our instruction compliance check suggested that most participants took the study seriously and followed instructions. Our sample included participants of varied ages, relationship statuses, and sexual identities, and these factors were meaningful covariates in our data, suggesting that they do play some role in effects of stimulus modality on arousal and desire. Future studies could explore these nuances; for example, might ISSE and fantasy conditions be especially effective for sexual minority participants? Additionally, future research could address how content and detail of imagined situations in the ISSE affect arousal and desire. Do participants who focus on their own pleasure in the imagined situation experience higher arousal, and can focusing on a partner's responses facilitate arousal as well?

\section{Conclusions}

Overall, our study provides experimental evidence that state desire responds to context in women and men, and that context influences the measurement of "trait" sexual desire in women. Together with others' findings, these results highlight problems with models that assume a linear progression from desire to arousal [9-11]. Our findings establish that solitary sexual desire can respond to context, and support understandings of responsive desire that include erotica and self-generated fantasy in addition to partner cues as triggers for desire. Additionally, our comparison of the ISSE with other modalities provides evidence for the utility of the ISSE in studies of sexual arousal and clarifies trade-offs of this method. Our findings suggest that fantasizing about self-selected sexually arousing content can elicit high levels of arousal compared with other modalities in women and men.

\section{Acknowledgments}

K.L.G. was supported by a National Science Foundation Graduate Research Fellowship (grant no. DGE0718128). We thank Lori Brotto, Meredith Chivers, and Caroline Pukall for helpful comments on an earlier draft of the manuscript, and William Chopik for statistical advice.

Corresponding Author: Sari M. van Anders, $\mathrm{PhD}$, Departments of Psychology \& Women's Studies, Program in Neuroscience, Reproductive Sciences Program, University of Michigan, 530 Church St., Ann Arbor, MI 48109, USA. Tel: 734-647-6981; Fax: 734764-3520; E-mail: smva@umich.edu

Conflict of Interest: None.

\section{Statement of Authorship}

\section{Category 1}

(a) Conception and Design

Katherine L. Goldey; Sari M. van Anders

(b) Acquisition of Data

Katherine L. Goldey

(c) Analysis and Interpretation of Data

Katherine L. Goldey; Sari M. van Anders

\section{Category 2}

(a) Drafting the Article

Katherine L. Goldey; Sari M. van Anders

(b) Revising It for Intellectual Content

Katherine L. Goldey; Sari M. van Anders

\section{Category 3}

(a) Final Approval of the Completed Article Katherine L. Goldey; Sari M. van Anders

\section{References}

1 Masters WH, Johnson VE. Human sexual response. Boston, MA: Little, Brown; 1966:366.

2 Kaplan HS. Hypoactive sexual desire. J Sex Marital Ther 1977;3:3-9. 
3 Kaplan HS. Disorders of sexual desire and other new concepts and techniques in sex therapy. New York: Brunner/Mazel; 1979:237.

4 Lief HI. Inhibited sexual desire. Med Aspects Hum Sex 1977;7:94-5.

5 Brotto LA. The DSM diagnostic criteria for Hypoactive Sexual Desire Disorder in women. Arch Sex Behav 2010;39:221-39.

6 Laan E, Both S. What makes women experience desire? Fem Psychol 2008;18:505-14.

7 Basson R. The female sexual response: A different model. J Sex Marital Ther 2000;26:51-65.

8 Both S, Everaerd E, Laan E. Desire emerges from excitement: A psychophysiological perspective on sexual motivation. In: Janssen E, ed. The psychophysiology of sex. Bloomington, IN: Indiana University Press; 2007:327-39.

9 Tiefer L. Historical, scientific, clinical, and feminist criticisms of "the human sexual response cycle" model. In: Tiefer L, ed. Sex is not a natural act and other essays. 2nd edition. Boulder, CO: Westview Press; 2004:41-61.

10 Tiefer L, Hall M, Tavris C. Beyond dysfunction: A new view of women's sexual problems. J Sex Marital Ther 2002; 28:225-32.

11 Hayes RD. Circular and linear modeling of female sexual desire and arousal. J Sex Res 2011;48:130-41.

12 Toates F. An integrative theoretical framework for understanding sexual motivation, arousal, and behavior. J Sex Res 2009;46:168-93.

13 Bancroft J, Graham CA. The varied nature of women's sexuality: Unresolved issues and a theoretical approach. Horm Behav 2011;59:717-29.

14 Brotto LA. The DSM diagnostic criteria for Hypoactive Sexual Desire Disorder in men. J Sex Med 2010;7:2015-30.

15 Janssen E, McBride KR, Yarber W, Hill BJ, Butler SM. Factors that influence sexual arousal in men: A focus group study. Arch Sex Behav 2008;37:252-65.

16 Meana M. Elucidating women's (hetero)sexual desire: Definitional challenges and content expansion. J Sex Res 2010;47:104-22.

17 van Anders SM, Brotto L, Farrell J, Yule M. Associations among physiological and subjective sexual response, sexual desire, and salivary steroid hormones in healthy premenopausal women. J Sex Med 2009;6:739-51.

18 Chivers ML, Bailey JM. A sex difference in features that elicit genital response. Biol Psychol 2005;70:115-20.

19 Payne KA, Binik YM, Pukall CF, Thaler L, Amsel R, Khalife S. Effects of sexual arousal on genital and non-genital sensation: A comparison of women with vulvar vestibulitis syndrome and healthy controls. Arch Sex Behav 2007;36:289_ 300 .

20 Heiman JR, Rupp H, Janssen E, Newhouse SK, Brauer M, Laan E. Sexual desire, sexual arousal and hormonal differences in premenopausal US and Dutch women with and without low sexual desire. Horm Behav 2011;59:772-9.

21 Heard-Davison A, Heiman JR, Kuffel S. Genital and subjective measurement of the time course effects of an acute dose of testosterone vs. placebo in postmenopausal women. J Sex Med 2007;4:209-17.

22 Peterson ZD, Janssen E. Ambivalent affect and sexual response: The impact of co-occurring positive and negative emotions on subjective and physiological sexual responses to erotic stimuli. Arch Sex Behav 2007;36:793-807.

23 Bozman AW, Beck JG. Covariation of sexual desire and sexual arousal: The effects of anger and anxiety. Arch Sex Behav 1991;20:47-60.

24 Graham CA, Janssen E, Sanders SA. Effects of fragrance on female sexual arousal and mood across the menstrual cycle. Psychophysiology 2000;37:76-84.
25 Toledano R, Pfaus J. The Sexual Arousal and Desire Inventory (SADI): A multidimensional scale to assess subjective sexual arousal and desire. J Sex Med 2006;3:853-77.

26 Both S, Spiering M, Everaerd W, Laan E. Sexual behavior and responsiveness to sexual stimuli following laboratory-induced sexual arousal. J Sex Res 2004;41:242-58.

27 Leitenberg H, Henning K. Sexual fantasy. Psychol Bull 1995;117:469-96.

28 Aslan G, Aslan D, Kizilyar A, Ispahi C, Esen A. A prospective analysis of sexual functions during pregnancy. Int J Impot Res 2005;17:154-7.

29 Klusmann D. Sexual motivation and the duration of partnership. Arch Sex Behav 2002;31:275-87.

30 Fisher WA, Rosen RC, Eardley I, Sand M, Goldstein I. Sexual experience of female partners of men with erectile dysfunction: The female experience of men's attitudes to life events and sexuality (FEMALES) study. J Sex Med 2005;2: 675-84.

31 Bodenmann G, Ledermann T, Blattner D, Galluzzo C. Associations among everyday stress, critical life events, and sexual problems. J Nerv Ment Dis 2006;194:494-501.

32 ter Kuile MM, Brauer M, Laan E. The Female Sexual Function Index (FSFI) and the Female Sexual Distress Scale (FSDS): Psychometric properties within a Dutch population. J Sex Marital Ther 2006;32:289-304.

33 Rosen R, Brown C, Heiman J, Leiblum S, Meston C, Shabsigh R, Ferguson D, D'Agostino R Jr. The Female Sexual Function Index (FSFI): A multidimensional self-report instrument for the assessment of female sexual function. J Sex Marital Ther 2000;26:191-208.

34 Spector IP, Carey MP, Steinberg L. The sexual desire inventory: Development, factor structure, and evidence of reliability. J Sex Marital Ther 1996;22:175-90.

35 Ariely D, Loewenstein G. The heat of the moment: The effect of sexual arousal on sexual decision making. J Behav Decis Mak 2006;19:87-98.

36 Goldhammer DL, McCabe MP. Development and psychometric properties of the Female Sexual Desire Questionnaire (FSDQ). J Sex Med 2011;8:2512-21.

37 van Anders SM, Hamilton LD, Schmidt N, Watson NV. Associations between testosterone secretion and sexual activity in women. Horm Behav 2007;51:477-82.

38 van Anders SM. Testosterone and sexual desire in healthy women and men. Arch Sex Behav 2012 May 3 [Epub ahead of print] doi: 10.1007/s10508-012-9946-2.

39 Heiman JR. Female sexual response patterns: Interactions of physiological, affective, and contextual cues. Arch Gen Psychiatry 1980;37:1311-6.

40 Heiman JR, Hatch JP. Affective and physiological dimensions of male sexual response to erotica and fantasy. Basic Appl Soc Psych 1980;1:315-27.

41 Julien E, Over R. Male sexual arousal across five modes of erotic stimulation. Arch Sex Behav 1988;17:131-43.

42 Koukounas E, Over R. Male sexual arousal elicited by film and fantasy matched in content. Aust J Psychol 1997;49:1-5.

43 Smith D, Over R. Correlates of fantasy-induced and filminduced male sexual arousal. Arch Sex Behav 1987;16:395409.

44 Laan E, Everaerd W, van Berlo R, Rijs L. Mood and sexual arousal in women. Behav Res Ther 1995;33:441-3.

45 Byrne D, Lamberth J. The effect of erotic stimuli on sex arousal, evaluative responses, and subsequent behavior. In: Technical report of the commission on obscenity and pornography. Vol. 8. Washington, DC: U.S. Government Printing Office; 1971:41-67.

46 Laan E, Everaerd W, van Bellen G, Hanewald G. Women's sexual and emotional responses to male- and female-produced erotica. Arch Sex Behav 1994;23:153-69. 
47 Goldey KL, van Anders SM. Sexy thoughts: Effects of sexual cognitions on testosterone, cortisol, and arousal in women. Horm Behav 2011;59:754-64.

48 Janssen E, Carpenter D, Graham CA. Selecting films for sex research: Gender differences in erotic film preference. Arch Sex Behav 2003;32:243-51.

49 Follingstad DR, Kimbrell CD. Sex fantasies revisited: An expansion and further clarification of variables affecting sex fantasy production. Arch Sex Behav 1986;15:475-86.

50 Dekker J, Everaerd W. Attentional effects on sexual arousal. Psychophysiology 1988;25:45-54.

51 Carlson ER, Coleman CE. Experiential and motivational determinants of the richness of an induced sexual fantasy. J Pers 1977;45:528-42.

52 Buhrmester M, Kwang T, Gosling SD. Amazon's Mechanical Turk: A new source of inexpensive, yet high-quality, data? Perspect Psychol Sci 2011;6:3-5.

53 van Anders SM, Goldey KL. Testosterone and partnering are linked via relationship status for women and "relationship orientation" for men. Horm Behav 2010;58:820-6.

54 Heiman JR, Rowland DL. Affective and physiological sexual response patterns: The effects of instructions on sexually functional and dysfunctional men. J Psychosom Res 1983;27: $105-16$.

55 Goldey KL, van Anders SM. Sexual thoughts: Links to testosterone and cortisol in men. Arch Sex Behav 2011 Oct 13 [Epub ahead of print] doi: 10.1007/s10508-011-9858-6.

56 Sorenson J. Set the dark on fire. New York: Bantam Books; 2009.

57 Prause N, Graham CA. Asexuality: Classification and characterization. Arch Sex Behav 2007;36:341-56.

58 Giargiari TD, Mahaffey AL, Craighead WE, Hutchison KE. Appetitive responses to sexual stimuli are attenuated in individuals with low levels of sexual desire. Arch Sex Behav 2005;34:547-56.

59 van Anders SM, Chernick AB, Chernick BA, Hampson E, Fisher WA. Preliminary clinical experience with androgen administration for pre- and postmenopausal women with hypoactive sexual desire. J Sex Marital Ther 2005;31:173-85.

60 Spector IP, Fremeth SM. Sexual behaviors and attitudes of geriatric residents in long-term care facilities. J Sex Marital Ther 1996;22:235-46.

61 King BE, Allgeier ER. The sexual desire inventory as a measure of sexual motivation in college students. Psychol Rep 2000;86:347-50.
62 van Anders SM, Dunn EJ. Are gonadal steroids linked with orgasm perceptions and sexual assertiveness in women and men? Horm Behav 2009;56:206-13.

63 Hayes RD, Dennerstein L, Bennett CM, Koochaki PE, Leiblum SR, Graziottin A. Relationship between Hypoactive Sexual Desire Disorder and aging. Fertil Steril 2007;87:10712.

64 Svedin CG, Akerman I, Priebe G. Frequent users of pornography: A population based epidemiological study of Swedish male adolescents. J Adolesc 2011;34:779-88.

65 Chivers ML, Rieger G, Latty E, Bailey JM. A sex difference in the specificity of sexual arousal. Psychol Sci 2004;15:736-44.

66 Garcia LT. Sex differences in sexual arousal to different erotic stories. J Sex Res 1984;20:391-402.

67 McCall KM, Rellini AH, Seal BN, Meston CM. Sex differences in memory for sexually-relevant information. Arch Sex Behav 2007;36:508-17.

68 Scott CL, Cortez A Jr. No longer his and hers, but ours: Examining sexual arousal in response to erotic stories designed for both sexes. J Sex Marital Ther 2011;37:165-75.

69 Revicki DA, Margolis MK, Bush EN, DeRogatis LR, Hanes V. Content validity of the Female Sexual Function Index (FSFI) in pre- and postmenopausal women with Hypoactive Sexual Desire Disorder. J Sex Med 2011;8:2237-45.

70 DeRogatis L, Pyke R, McCormack J, Hunter A, Harding G. Does the Female Sexual Distress Scale-Revised cover the feelings of women with HSDD? J Sex Med 2011;8:2810-5.

71 Basson R. Biopsychosocial models of women's sexual response: Applications to management of "desire disorders". Sex Relat Ther 2003;18:107-15.

72 Brotto LA, Heiman JR, Tolman DL. Narratives of desire in mid-age women with and without arousal difficulties. J Sex Res 2009;46:387-98.

73 Graham CA, Sanders SA, Milhausen RR, McBride KR. Turning on and turning off: A focus group study of the factors that affect women's sexual arousal. Arch Sex Behav 2004;33:527-38.

74 Beck JG, Bozman A, Qualtrough T. The experience of sexual desire: Psychological correlates in a college sample. J Sex Res 1991;28:443-56.

75 Carvalheira AA, Brotto LA, Leal I. Women's motivations for sex: Exploring the diagnostic and statistical manual, fourth edition, text revision criteria for hypoactive sexual desire and female sexual arousal disorders. J Sex Med 2010; $7: 1454-63$. 